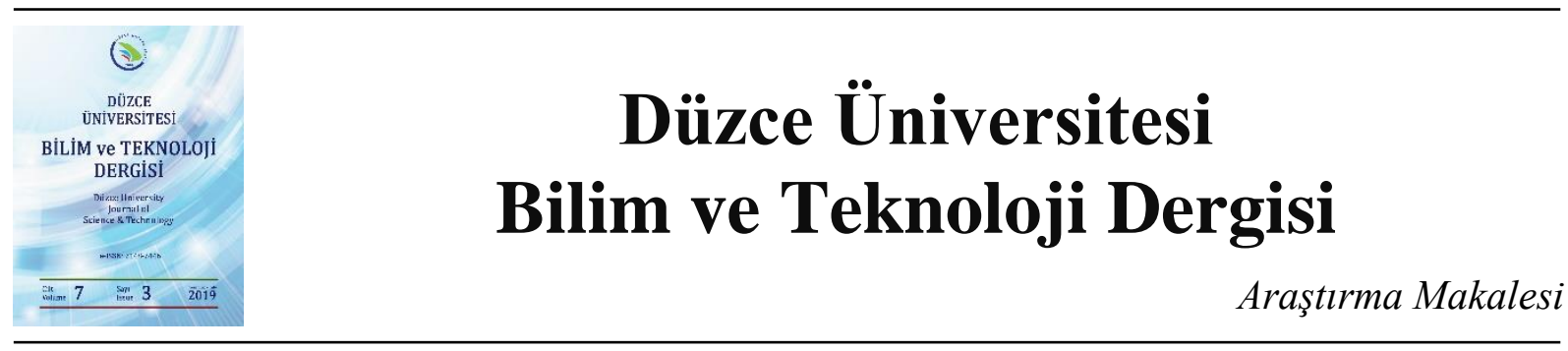

\section{Odun Yüzey Pürüzlülüğü Tahmininde Bir Yapay Sinir Ağı Modelinin Kullanılması}

\author{
(iD) Şükrü ÖZŞAHİN ${ }^{a^{*}}$, (iD) Hilal SINGER ${ }^{\mathrm{b}}$ \\ ${ }^{a}$ Endüstri Mühendisliği Bölümü, Mühendislik Fakültesi, Karadeniz Teknik Üniversitesi, Trabzon, TÜRKIYYE \\ ${ }^{b}$ Endüstri Mühendisliği Bölümü, Mühendislik Fakültesi, Bolu Abant İzzet Baysal Üniversitesi, Bolu, TÜRKIYYE \\ * Sorumlu yazarin e-posta adresi: sukru@ktu.edu.tr \\ DOI : 10.29130/dubited.554419
}

\begin{abstract}
ÖZET
Ağaç malzemelerin yüzey pürüzlülüğü, nihai ürünlerin kalitesinin değerlendirilmesi açısından çok önemlidir. Bu nedenle bu çalışmada, odun türü, bıçak sayısı, besleme hızı ve kesme derinliğinin planyalama işleminde yüzey pürüzlülüğü üzerindeki etkisini modellemek için bir yapay sinir ağı (YSA) modeli geliştirilmiştir. Farklı YSA modelleri oluşturulmuş ve bunların performansı ortalama mutlak yüzde hata (MAPE), ortalama karesel hatanın karekökü (RMSE) ve determinasyon katsayısı $\left(\mathrm{R}^{2}\right)$ kullanılarak değerlendirilmiştir. Önerilen modelin test safhasındaki MAPE, RMSE ve $\mathrm{R}^{2}$ değerleri sırasıyla \%7,27, 0,57 ve 0,903 olmuştur. Sonuç olarak YSA, planyalanan odunun yüzey pürüzlülüğünü tahmin etmede etkili bir araçtır ve maliyetli ve zaman alıcı araştırmalar yerine oldukça yararlıdır.
\end{abstract}

Anahtar Kelimeler: Yapay sinir ăğ, Yüzey pürüzlülü̆̆̈̈, Bıçak sayısl, Besleme hızı, Kesme derinliği

\section{Utilizing an Artificial Neural Network Model in Wood Surface Roughness Prediction}

\begin{abstract}
The surface roughness of wood materials is very important in terms of assessing the quality of final products. Therefore, in this study, an artificial neural network (ANN) model was developed to model the effect of wood species, number of knives, feed rate, and cutting depth on surface roughness in the planing process. Different ANN models were created and the performance of them was evaluated using the mean absolute percentage error (MAPE), the root mean square error (RMSE), and the coefficient of determination $\left(\mathrm{R}^{2}\right)$. The MAPE, RMSE, and $\mathrm{R}^{2}$ values in the testing phase of the proposed model were $7.27 \%, 0.57$, and 0.903 , respectively. Consequently, ANN is an effective tool in predicting the surface roughness of planed wood and quite useful instead of costly and time-consuming investigations.
\end{abstract}

Keywords: Artificial neural network, Surface roughness, Number of knives, Feed rate, Cutting depth 


\section{GiRISS}

A ğaç malzeme, işlenme kolaylığı, işlenme sırasında düşük enerji tüketimi gereksinimi, farklı renk ve desenlerde bulunabilirliği, ses ve 1sı iletim özellikleri ile yüzey işlemleri için uygunluğu gibi özellikleri nedeniyle iç ve dış dekorasyonda yaygın olarak kullanılmaktadır [1]. Masif ağaç malzeme, biçme, planyalama, zımparalama vb. işlemlerden geçirildikten sonra nihai bir ürün haline gelir [2]. Odunun ve kesme aletinin arasındaki etkileşimin sonucu olarak yüzey üzerindeki büyüklüğg̈ işleme kalitesini karakterize eden düzensizlikler ortaya çıkmaktadır. Üretim sürecinin veya malzeme durumunun neden olduğu bu yüzey düzensizlikleri pürüzlülük olarak tanımlanabilir [3].

Ahşap ve ahşap-esaslı ürünlerin yüzey kalitesi genellikle yüzey düzensizlikleri ile belirlenir. Düzensizlikler, yüzey pürüzlülüğü olarak değerlendirilmekte olup bunların yüksekliği, genişliği ve şekli bir ürünün yüzey kalitesini belirler [4]. Yüzey pürüzlülüğünün kontrol edilmesi ve izlenmesi ürün kalitesinin üretim boyunca aynı seviyede tutulması için gereklidir. Ayrıca, yüzey pürüzlülüğü üretimin ileriki safhalarını olumsuz yönde etkileyebilmektedir [2].

Niteliksel ve niceliksel olarak yüzey pürüzlülüğü değerlendirilebilir. Yüzeyin niteliksel olarak değerlendirilmesinde görsel ve dokunsal yöntemler kullanılabilir. Ancak, bu yöntemler ile yapılan değerlendirmeler insanın öznel algısına dayanmaktadır. Başka bir deyişle, hem görsel hem de dokunsal değerlendirmeler ile sadece brüt karşılaştırmaların yapılması mümkün olup odunun yüzey özellikleri hakkında spesifik bilgi elde edilememektedir. Yüzey kalitesinin objektif ve niceliksel olarak değerlendirilmesi için ölçüm araçları kullanılmalı ve yüzey pürüzlülüğü sayısal değerler ile ifade edilmelidir. $\mathrm{Bu}$ amaçla, dokunmalı iğne tarama, optik, ultrasonik ve video kamera kullanımı ile görüntü analizi gibi yöntemler kullanılabilir [3,5].

Yüzey kalitesinin sayısal değerlendirilmesi farklı yüzey dokuları arasında karşılaştırmaların yapılmasına imkân veren pürüzlülük parametrelerinin hesaplanmasını gerekli kılar [3]. Yüzey pürüzlülüğü parametreleri, bir yüzeyin iki boyutlu profilini profil ortalama çizgisine göre vermekle birlikte girintilerin ve çıkıntıların meydana getirdiği düzensizlikleri de göstermektedir [6]. Yüzey pürüzlülüğü ölçümü tarihinde (1930'lardan beri) sayısız yüzey pürüzlülüğü parametresi önerilmiş ve bunların birçoğu uluslararası ve ulusal standartlar olarak tanıtılmıştır. Bu parametreler arasından en çok kullanılanı ortalama pürüzlülük (Ra) tür [7]. Ra, örnek uzunluğu içerisindeki profil ortalama çizgisinden sapmalar ile ilgili değerlerin aritmetik ortalamasının alınmasıdır [6].

Ürünlerin kalitesinin belirlenmesinde önemli bir kıstas olan yüzey pürüzlülüğü, işlemenin ve odun yapısının bir kombinasyonudur. Çünkü işleme tabi tutulan odunun yüzey kalitesi hem işleme koşulları (kesme hızı, kesme derinliği, kullanılan bıçağın körlüğü, kesme açısı, besleme hızı vb.) hem de odun özellikleriyle (tür, yoğunluk, ilkbahar-yaz odunu oranı, rutubet miktarı vb.) ilgili birçok faktörden etkilenmektedir [7]. Bu sebeple, yüksek kaliteli bir yüzeye ulaşmak için işleme koşullarına ve odun özelliklerine ilişkin faktörlerin değerlendirilmesi önemlidir.

Nihai ürünün yüzey kalitesini iyileştirmek için son yıllarda ahşap ve ahşap-esaslı malzemelerin yüzey pürüzlülüğü ile ilgili araştırma çalışmaları artış göstermiştir [1,2, 8-11]. Bu çalışmalar göstermiştir ki, daha iyi bir yüzey kalitesi elde etmek için uygun işleme koşullarının seçimi önemlidir. Ancak, proses parametrelerinin optimum değerlerini belirlemek için yapılan kapsamlı testler yüksek maliyetlere ve zaman kaybına neden olabilmektedir. Modelleme yöntemleri parametrelerin uygun kombinasyonlarını belirlemek için kullanılabilir. Bu sayede, parametrelerin etkilerinin araştırılması için gerekli olan test 
sayısı azaltılabilir. Son yıllarda, yapay sinir ağı (YSA) modelleme yaklaşımının değişkenler arasındaki ilişkiyi taklit etme kabiliyeti birçok araştırmacının dikkatini çekmiştir [12]. Ahşap bilimi alanındaki bazı çalışmalar da çeşitli sorunları çözmek için YSA yaklaşımını kullanmıştır [13-17].

Literatürde, çeşitli parametrelerin odunun yüzey pürüzlülügü üzerine olan etkileri detaylı olarak ele alınmıştır. Ancak, odunun yüzey pürüzlülügünün tahmini ile ilgili çalışmaların sayısı oldukça azdır [17]. $\mathrm{Bu}$ nedenle, bu çalışmanın amacı odun türü, bıçak sayısı, besleme hızı ve kesme derinliği değişkenlerinin planyalama ile oluşan yüzey pürüzlülüğü üzerine olan etkilerini modellemek için bir YSA modelinin tasarlanması ve geliştirilen YSA modelini kullanarak bahsi geçen proses parametrelerinin optimize edilmesidir.

\section{Materyal Ve Metot}

\section{A. VERI TOPLAMA}

$\mathrm{Bu}$ çalışmada kullanılan veriler Demirci [10] tarafından yapılan bir çalışmadan elde edilmiştir. Yazar, sarıçam (Pinus sylvestris L.), kayın (Fagus orientalis L.), ceviz (Juglans regia L.), kestane (Castanea sativa M.) ve mahun (Khaya sp.) odunlarını deney materyalleri olarak seçmiştir. Deney numunelerini 1,2 ve $4 \mathrm{~mm}$ kesme derinliğinde, 5 ve $9 \mathrm{~m} / \mathrm{dk}$ besleme hızında ve 2 ve 4 bıçak kullanarak planyalamış ve yüzey pürüzlülüğü değerlerini Mitutoyo Sj-301 cihazı ile ölçmüştür. Numunelerin pürüzlülük sonuçlarının değerlendirilmesinde Ra parametresini kullanmıştır.

\section{B. YAPAY SINIR A $\breve{G L A R I ~}$}

YSA'lar, karmaşık sorunlarla başa çıkmak için etkili çözümler sunan veri modelleme araçlarıdır. Değişkenler arasındaki karmaşık ve doğrusal olmayan ilişkileri öğrenme yeteneklerinden dolayı tahmin, sınıflandırma, optimizasyon, kümeleme ve simülasyon gibi birçok uygulamada yaygın olarak kullanılmaktadırlar [18].

Tahmin amaçlı en yaygın kullanılan YSA tipi çok katmanlı algılayıcı (ÇKA) dır. ÇKA'nın yapısı üç farklı katmandan oluşur: (1) verinin ağa sunulduğu girdi katmanı, (2) girdi katmanından gelen bilgilerin işlendiği gizli katman ve (3) sonuçların elde edildiği çıktı katmanı [19]. Şekil 1, ÇKA mimarisinin tipik bir örneğini göstermektedir [16].

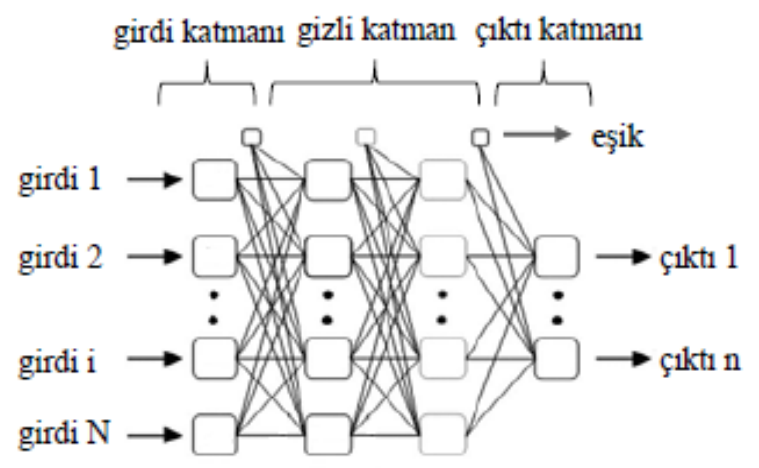

Şekil 1. Bir ÇKA örneği 
İşlemci elemanlar (nöronlar), YSA katmanlarındaki temel öğedir. Girdi nöronları ve çıtı nöronları sırasıyla girdileri ve çıktıları temsil eder. Ayrıca, ele alınan problemin karmaşıklık seviyesine bağlı olarak değişebilen gizli nöronlar da bulunmaktadır [20]. ÇKA'daki nöronlar birbirlerine ağırlıklı bağlantılar ile bağlanır, ancak bu bağlantılar aynı katman içinde bulunmaz. Herhangi bir nöron, eşik ve ağırlıklı giriş sinyallerini toplar, toplamı bir aktivasyon fonksiyonu işler ve sonucu sonraki katmanın nöronlarına gönderir [16].

Sinir ağı, değişkenler arasındaki ilişkiyi öğrenmek için eğitilir. Eğitim, istenen çıktıyı elde etmek için ağ katmanlarında bulunan nöronların arasındaki bağlantının en iyi ağırlıklarını bulmaktır. Hata seviyesi hedeflenen değere ulaştığında ağın eğitimi biter ve böylece bir model elde edilir. Ardından, modelin performansı eğitimin herhangi bir aşamasında kullanılmamış bir veri seti ile değerlendirilir [17].

\section{YAPAY SINIR A ĞI ANALIZI}

$\mathrm{Bu}$ çalışma, odunun yüzey pürüzlülüğü değerlerinin YSA yaklaşımı ile tahminine odaklanmıştır. Ağların inşa edilmesinde kullanılan veriler literatürdeki deneysel bir çalışmadan elde edilmiştir [10]. YSA modellerinde, odun türü, bıçak sayısı, besleme hızı ve kesme derinliği değişkenleri girdi olarak dikkate alınırken odunun yüzey pürüzlülüğü (Ra) çıktı olarak kullanılmıştır. YSA modelleri, MATLAB paket programı kullanılarak oluşturulmuştur.

Odun türü, bıçak sayısı, besleme hızı ve kesme derinliği değişkenlerinin yüzey pürüzlülüğü üzerindeki etkilerini incelemek için deney verileri (60 veri) eğitim, doğrulama ve test verileri şeklinde rastgele ve homojen bir biçimde gruplandırılmıştır. YSA'ların eğitimi için 40 veri (tümünün \%66,66's1) kullanılmıştır. Geriye kalan 20 veri, doğrulama (tümünün \%16,67'si) ve test (tümünün \%16,67'si) için eşit olarak bölünmüştür. Çalışmada kullanılan veri seti Tablo 1'de verilmiştir.

Tablo 1. Çalışmada kullanılan veri seti

\begin{tabular}{lllll}
\hline \multicolumn{1}{c}{ Odun türü } & Bıçak sayısı & Besleme hızı & Kesme derinliği & $\mathbf{R}_{\mathbf{a}}$ \\
\hline Pinus sylvestris L. & $\mathbf{2}$ & $\mathbf{5}$ & $\mathbf{1}$ & $\mathbf{3 , 5 5}$ \\
\hline Pinus sylvestris L. & 2 & 5 & 2 & 3,63 \\
\hline Pinus sylvestris L. & 2 & 5 & 1 & 3,76 \\
\hline Pinus sylvestris L. & 2 & 9 & $\mathbf{2}$ & 3,75 \\
\hline Pinus sylvestris L. & $\mathbf{2}$ & $\mathbf{9}$ & 4 & $\mathbf{3 , 9 8}$ \\
\hline Pinus sylvestris L. & 2 & 9 & 1 & 3,99 \\
\hline Pinus sylvestris L. & 4 & 5 & 2 & 2,87 \\
\hline Pinus sylvestris L. & 4 & 5 & $\mathbf{4}$ & 3,19 \\
\hline Pinus sylvestris L. & $\mathbf{4}$ & $\mathbf{5}$ & $\mathbf{3 , 6 4}$ \\
\hline Pinus sylvestris L. & 4 & 9 & $\mathbf{2}$ & 3,04 \\
\hline Pinus sylvestris $\boldsymbol{L}$. & $\mathbf{4}$ & $\mathbf{9}$ & $\mathbf{3 , 5 3}$ \\
\hline Pinus sylvestris L. & 4 & 9 & 1 & 3,74 \\
\hline Fagus orientalis L. & 2 & 5 & 2 & 3,87 \\
\hline Fagus orientalis L. & 2 & 5 & $\mathbf{4}$ & 4,19 \\
\hline Fagus orientalis $\boldsymbol{L}$. & $\mathbf{2}$ & $\mathbf{5}$ & $\mathbf{1}, \mathbf{0 8}$ \\
\hline Fagus orientalis L. & $\mathbf{2}$ & $\mathbf{9}$ & $\mathbf{4 , 6 7}$ \\
\hline
\end{tabular}


Tablo 1. (devam). Çalışmada kullanılan veri seti

$\begin{array}{lllll}\text { Odun türü } & \text { Bıçak sayısı } & \text { Besleme hızı } & \text { Kesme derinliği } & \mathbf{R}_{\mathrm{a}}\end{array}$

\begin{tabular}{|c|c|c|c|c|}
\hline Fagus orientalis L. & 2 & 9 & 2 & 5,61 \\
\hline Fagus orientalis L. & 2 & 9 & 4 & 6,31 \\
\hline Fagus orientalis L. & 4 & 5 & 1 & 3,55 \\
\hline Fagus orientalis $L$. & 4 & 5 & 2 & 3,96 \\
\hline Fagus orientalis L. & 4 & 5 & 4 & 4,02 \\
\hline Fagus orientalis L. & 4 & 9 & 1 & 4,07 \\
\hline Fagus orientalis L. & 4 & 9 & 2 & 4,30 \\
\hline Fagus orientalis L. & 4 & 9 & 4 & 4,98 \\
\hline Juglans regia L. & 2 & 5 & 1 & 5,35 \\
\hline Juglans regia L. & 2 & 5 & 2 & 5,56 \\
\hline Juglans regia L. & 2 & 5 & 4 & 6,56 \\
\hline Juglans regia L. & 2 & 9 & 1 & 6,72 \\
\hline Juglans regia L. & 2 & 9 & 2 & 7,13 \\
\hline Juglans regia $L$. & 2 & 9 & 4 & 6,80 \\
\hline Juglans regia $\mathbf{L}$. & 4 & 5 & 1 & 3,72 \\
\hline Juglans regia L. & 4 & 5 & 2 & 5,82 \\
\hline Juglans regia L. & 4 & 5 & 4 & 6,08 \\
\hline Juglans regia L. & 4 & 9 & 1 & 4,15 \\
\hline Juglans regia $L$. & 4 & 9 & 2 & 6,71 \\
\hline Juglans regia L. & 4 & 9 & 4 & 6,71 \\
\hline Castanea sativa M. & 2 & 5 & 1 & 7,49 \\
\hline Castanea sativa M. & 2 & 5 & 2 & 6,16 \\
\hline Castanea sativa $M$. & 2 & 5 & 4 & 6,62 \\
\hline Castanea sativa M. & 2 & 9 & 1 & 7,57 \\
\hline Castanea sativa M. & 2 & 9 & 2 & 6,32 \\
\hline Castanea sativa M. & 2 & 9 & 4 & 6,57 \\
\hline Castanea sativa $M$. & 4 & 5 & 1 & 6,48 \\
\hline Castanea sativa M. & 4 & 5 & 2 & 9,77 \\
\hline Castanea sativa M. & 4 & 5 & 4 & 5,21 \\
\hline Castanea sativa M. & 4 & 9 & 1 & 6,55 \\
\hline Castanea sativa M. & 4 & 9 & 2 & 8,81 \\
\hline Castanea sativa M. & 4 & 9 & 4 & 6,09 \\
\hline Khaya sp. & 2 & 5 & 1 & 5,83 \\
\hline Khaya sp. & 2 & 5 & 2 & 7,19 \\
\hline Khaya sp. & 2 & 5 & 4 & 8,32 \\
\hline Khaya sp. & 2 & 9 & 1 & 7,97 \\
\hline Khaya sp. & 2 & 9 & 2 & 10,01 \\
\hline Khaya sp. & 2 & 9 & 4 & 7,99 \\
\hline Khaya sp. & 4 & 5 & 1 & 5,56 \\
\hline Khaya sp. & 4 & 5 & 2 & 6,86 \\
\hline Khaya sp. & 4 & 5 & 4 & 7,18 \\
\hline Khaya sp. & 4 & 9 & 1 & 6,35 \\
\hline Khaya sp. & 4 & 9 & 2 & 7,22 \\
\hline Khaya sp. & 4 & 9 & 4 & 7,81 \\
\hline
\end{tabular}


Farklı ağ yapılarına ve parametrelerine (aktivasyon fonksiyonu, eğitim algoritması, öğrenme kuralı vb.) sahip birçok YSA modeli oluşturulmuştur. Eğitim safhası, en uygun ağırlık ve eşik değerlerinin belirlenmesi için gerçekleştirilmiştir. Doğrulama safhası, modelin performansını izlemek için kullanılmıştır. Modelin genelleme yeteneğini değerlendirmek için test safhası gerçekleştirilmiştir. Sonuç olarak; ilk katmanında 2, ikinci katmanında 6 nöron bulunan iki gizli katmana sahip ağ mimarisi (bkz. Şekil 2) tahminler yapmak için seçilmiştir.

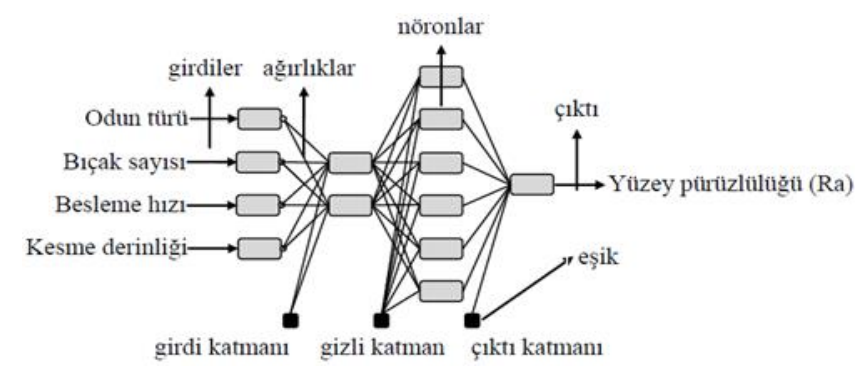

Şekil 2. Tahmin modelinin mimarisi

Tahmin modelinin belirlenmesi için ileri beslemeli ve geri yayılımlı çok katmanlı YSA tercih edilmiştir. Modelin gizli katmanlarında hiperbolik tanjant sigmoid fonksiyonu, çıktı katmanında ise doğrusal transfer fonksiyonu aktivasyon fonksiyonu olarak kullanılmıştır. Levenberg-Marquardt algoritması eğitim algoritması olarak seçilmiş, momentumlu gradyan azaltım geri yayılım algoritması öğrenme kuralı olarak kullanılmış ve ortalama karesel hata (MSE) performans fonksiyonu olarak tercih edilmiştir. MSE, Eşt. 1 kullanılarak hesaplanmıştır. Eşitlikte $t_{i}$, gerçek değeri; $t d_{i}$, tahmin edilen değeri ve $N$, toplam örnek sayısını temsil etmektedir.

$\operatorname{MSE}=\frac{1}{N} \sum_{i=1}^{N}\left(t_{i}-t d_{i}\right)^{2}$

Parametrelerin önemini denk hale getirmek için eğitim, doğrulama ve test safhalarından önce verilerin normalleştirilmesi önerilmektedir [21]. Modelde hiperbolik tanjant sigmoid fonksiyonunun kullanılması nedeniyle çalışmanın tüm parametreleri [-1 1] aralığındaki bir sayı ile eşleştirilmiştir. Model çıktıları ters normalize işlemi sayesinde orijinal değerlere dönüştürülmüştür. Normalizasyon Eşt. 2'nin kullanımıyla gerçekleştirilmiştir. Eşitlikte $X_{\text {norm }}$, normalize edilmiş veriyi; $X$, değişkenin gerçek değerini; $X_{\min }$, veri grubunun minimum değerini ve $X_{\max }$, veri grubunun maksimum değerini ifade etmektedir.

$X_{\text {norm }}=2 \times \frac{X-X_{\min }}{X_{\max }-X_{\min }}-1$

Oluşturulan YSA modellerini karşılaştırmak ve hangi YSA modelinin en iyi olduğuna karar vermek için ortalama mutlak yüzde hata (MAPE), ortalama karesel hatanın karekökü (RMSE) ve determinasyon katsayısı $\left(\mathrm{R}^{2}\right)$ kullanılmıştır. MAPE, RMSE ve $\mathrm{R}^{2}$ değerleri sırasıyla Eşt. 3, Eşt. 4 ve Eşt. 5 ile hesaplanmıştır. Eşt. 5 'teki $\bar{t}$, tahmini değerlerin ortalamasını temsil etmektedir. 


$$
\begin{aligned}
& \text { MAPE }=\frac{1}{N}\left(\sum_{i=1}^{N}\left[\left|\frac{t_{i}-t d_{i}}{t_{i}}\right|\right]\right) \times 100 \\
& \mathrm{RMSE}=\sqrt{\frac{1}{N} \sum_{i=1}^{N}\left(t_{i}-t d_{i}\right)^{2}} \\
& \mathrm{R}^{2}=1-\frac{\sum_{i=1}^{N}\left(t_{i}-t d_{i}\right)^{2}}{\sum_{i=1}^{N}\left(t_{i}-\bar{t}\right)^{2}}
\end{aligned}
$$

\section{BULGULAR VE TARTIȘMA}

Odun türü, bıçak sayısı, besleme hızı ve kesme derinliği değişkenlerinin yüzey pürüzlülüğü üzerine olan etkilerini modellemek için YSA modeli geliştirilmiştir. Gerçek değerler, model çıktıları, yüzde hatalar ve MAPE ve RMSE değerleri eğitim için Tablo 2'de, doğrulama için Tablo 3'te, test için Tablo 4'te verilmiştir.

Tablo 2. Eğitim veri seti ve tahmin modeli sonuçları

\begin{tabular}{lcccccc}
\hline \multirow{2}{*}{ Odun türüi } & \multirow{2}{*}{ Bıçak sayısı } & \multirow{2}{*}{ Besleme hızı } & \multirow{2}{*}{ Kesme derinliği } & \multicolumn{3}{c}{ Ra } \\
\cline { 5 - 7 } & & & & Gerçek & Tahmin & Hata (\%) \\
\hline Pinus sylvestris L. & 2 & 5 & 2 & 3,63 & 3,48 & 4,14 \\
\hline Pinus sylvestris L. & 2 & 5 & 4 & 3,76 & 3,63 & 3,49 \\
\hline Pinus sylvestris L. & 2 & 9 & 1 & 3,75 & 3,86 & $-2,88$ \\
\hline Pinus sylvestris L. & 2 & 9 & 4 & 3,99 & 4,28 & $-7,28$ \\
\hline Pinus sylvestris L. & 4 & 5 & 1 & 2,87 & 3,29 & $-14,51$ \\
\hline Pinus sylvestris L. & 4 & 5 & 2 & 3,19 & 3,32 & $-3,97$ \\
\hline Pinus sylvestris L. & 4 & 9 & 1 & 3,04 & 3,29 & $-8,37$ \\
\hline Pinus sylvestris L. & 4 & 9 & 4 & 3,74 & 3,51 & 6,02 \\
\hline Fagus orientalis L. & 2 & 5 & 1 & 3,87 & 3,92 & $-1,28$ \\
\hline Fagus orientalis L. & 2 & 5 & 2 & 4,19 & 4,10 & 2,16 \\
\hline Fagus orientalis L. & 2 & 9 & 2 & 5,61 & 5,31 & 5,37 \\
\hline Fagus orientalis L. & 2 & 9 & 4 & 6,31 & 6,09 & 3,48 \\
\hline Fagus orientalis L. & 4 & 5 & 1 & 3,55 & 3,48 & 1,84 \\
\hline Fagus orientalis L. & 4 & 5 & 4 & 4,02 & 4,50 & $-11,85$ \\
\hline Fagus orientalis L. & 4 & 9 & 1 & 4,07 & 3,53 & 13,18 \\
\hline Fagus orientalis L. & 4 & 9 & 2 & 4,30 & 3,76 & 12,65 \\
\hline Juglans regia L. & 2 & 5 & 1 & 5,35 & 5,44 & $-1,59$ \\
\hline Juglans regia L. & 2 & 5 & 4 & 6,56 & 6,73 & $-2,59$ \\
\hline Juglans regia L. & 2 & 9 & 1 & 6,72 & 6,90 & $-2,63$ \\
\hline Juglans regia L. & 2 & 9 & 2 & 7,13 & 7,24 & $-1,56$ \\
\hline Juglans regia L. & 4 & 5 & 2 & 5,82 & 5,23 & 10,11 \\
\hline Juglans regia L. & 4 & 5 & 4 & 6,08 & 6,10 & $-0,32$ \\
\hline Juglans regia L. & 4 & 9 & 1 & 4,15 & 4,77 & $-15,05$ \\
\hline Juglans regia L. & 4 & 9 & 4 & 6,71 & 6,80 & $-1,39$ \\
& & & & & & 1770
\end{tabular}


Tablo 2. (devam). Eğitim veri seti ve tahmin modeli sonuçları

\begin{tabular}{ccccccc} 
Castanea sativa M. & 2 & 5 & 1 & 7,49 & 7,15 & 4,53 \\
\hline Castanea sativa M. & 2 & 5 & 2 & 6,16 & 6,35 & $-3,01$ \\
\hline Castanea sativa M. & 2 & 9 & 1 & 7,57 & 7,51 & 0,79 \\
\hline Castanea sativa M. & 2 & 9 & 4 & 6,57 & 6,60 & $-0,39$ \\
\hline Castanea sativa M. & 4 & 5 & 2 & 9,77 & 9,78 & $-0,12$ \\
\hline Castanea sativa M. & 4 & 5 & 4 & 5,21 & 5,26 & $-0,87$ \\
\hline Castanea sativa M. & 4 & 9 & 2 & 8,81 & 8,82 & $-0,13$ \\
\hline Castanea sativa M. & 4 & 9 & 4 & 6,09 & 6,06 & 0,42 \\
\hline Khaya sp. & 2 & 5 & 2 & 7,19 & 7,18 & 0,09 \\
\hline Khaya sp. & 2 & 5 & 4 & 8,32 & 8,33 & $-0,06$ \\
\hline Khaya sp. & 2 & 9 & 1 & 7,97 & 7,97 & 0,05 \\
\hline Khaya sp. & 2 & 9 & 2 & 10,01 & 9,99 & 0,25 \\
\hline Khaya sp. & 4 & 5 & 1 & 5,56 & 5,58 & $-0,37$ \\
\hline Khaya sp. & 4 & 5 & 4 & 7,18 & 7,31 & $-1,82$ \\
\hline Khaya sp. & 4 & 9 & 1 & 6,35 & 6,28 & 1,14 \\
\hline Khaya sp. & 4 & 9 & 2 & 7,22 & 7,11 & 1,51 \\
\hline MAPE $=\mathbf{3 , 8 3}$ & & & & & $\mathbf{R M S E}=\mathbf{0 , 2 5}$ \\
\hline
\end{tabular}

Tablo 3. Doğrulama veri seti ve tahmin modeli sonuçları

\begin{tabular}{ccccccc}
\hline \multirow{2}{*}{ Odun türü } & \multirow{2}{*}{ Bıçak sayısı } & \multirow{2}{*}{ Besleme hızı } & \multirow{2}{*}{ Kesme derinliği } & \multicolumn{3}{c}{ Ra } \\
\cline { 5 - 8 } & & & Gerçek & Tahmin & Hata (\%) \\
\hline Pinus sylvestris L. & 2 & 5 & 1 & 3,55 & 3,44 & 3,22 \\
\hline Pinus sylvestris L. & 4 & 5 & 4 & 3,64 & 3,47 & 4,69 \\
\hline Fagus orientalis L. & 2 & 9 & 1 & 4,67 & 5,02 & $-7,55$ \\
\hline Fagus orientalis L. & 4 & 9 & 4 & 4,98 & 4,70 & 5,65 \\
\hline Juglans regia L. & 2 & 5 & 2 & 5,56 & 5,97 & $-7,37$ \\
\hline Juglans regia L. & 4 & 5 & 1 & 3,72 & 4,57 & $-22,77$ \\
\hline Castanea sativa M. & 2 & 9 & 2 & 6,32 & 6,45 & $-2,09$ \\
\hline Castanea sativa M. & 4 & 9 & 1 & 6,55 & 7,20 & $-9,96$ \\
\hline Khaya sp. & 2 & 5 & 1 & 5,83 & 5,71 & 2,09 \\
\hline Khaya sp. & 4 & 5 & 2 & 6,86 & 6,98 & $-1,75$ \\
\hline MAPE $=\mathbf{6 , 7 1}$ & & & & & RMSE $=\mathbf{0 , 4 0}$ \\
\hline
\end{tabular}

Tablo 4. Test veri seti ve tahmin modeli sonuçları

\begin{tabular}{ccccccc}
\hline \multirow{2}{*}{ Odun türü } & \multirow{2}{*}{ Bıçak sayısı } & \multirow{2}{*}{ Besleme hızı } & \multirow{2}{*}{ Kesme derinliği } & \multicolumn{3}{c}{ Ra } \\
\cline { 5 - 8 } & & & Gerçek & Tahmin & Hata (\%) \\
\hline Pinus sylvestris L. & 2 & 9 & 2 & 3,98 & 3,97 & 0,29 \\
\hline Pinus sylvestris L. & 4 & 9 & 2 & 3,53 & 3,33 & 5,65 \\
\hline Fagus orientalis L. & 2 & 5 & 4 & 5,08 & 4,74 & 6,69 \\
\hline Fagus orientalis L. & 4 & 5 & 2 & 3,96 & 3,67 & 7,30 \\
\hline Juglans regia L. & 2 & 9 & 4 & 6,80 & 7,32 & $-7,59$ \\
\hline Juglans regia L. & 4 & 9 & 2 & 6,71 & 5,42 & 19,17 \\
\hline Castanea sativa M. & 2 & 5 & 4 & 6,62 & 6,09 & 8,00 \\
\hline Castanea sativa M. & 4 & 5 & 1 & 6,48 & 6,29 & 2,95 \\
\hline Khaya sp. & 2 & 9 & 4 & 7,99 & 8,70 & $-8,83$ \\
\hline
\end{tabular}


Tablo 4 (devam). Test veri seti ve tahmin modeli sonuçları

\begin{tabular}{ccccccc}
\hline Khaya sp. & 4 & 9 & 4 & 7,81 & 7,32 & 6,25 \\
\hline MAPE $=\mathbf{7 , 2 7}$ & & & & & \multicolumn{2}{c}{ RMSE $=\mathbf{0 , 5 7}$} \\
\hline
\end{tabular}

MAPE, en önemli değerlendirme kriterlerinden biridir ve birçok araştırmacı MAPE'yi kullanarak model performansını değerlendirmiştir [22-24]. MAPE'nin yüzey pürüzlülüğü tahmin modeli için eğitim, doğrulama ve test safhalarında hesaplanan değerleri sırasılla $\% 3,83, \% 6,71$ ve $\% 7,27$ olmuştur. Literatürde, MAPE değerinin \%10'un altına olması durumunda model performansının yüksek olduğu belirtilmiştir [24]. Bu bilginin 1şığında, önerilen YSA modelinin tahmin yeteneğinin mükemmel olduğu söylenebilir.

RMSE’nin yüzey pürüzlülüğü tahmin modeli için eğitim safhasındaki değeri 0,25 , doğrulama safhasındaki değeri 0,40 ve test safhasındaki değeri 0,57 olmuştur. RMSE değerlerinin düşük olması model performansının iyi olduğunu gösterir [25]. Bu çalışmada hesaplanan RMSE sonuçlarına dayanarak, yapılan tahminlerin başarılı olduğu söylenebilir.

$\mathrm{R}^{2}$ kriteri, gerçek değerler ile model çıktıları arasındaki ilişkinin seviyesini göstermek için yaygın olarak kullanılmaktadır. Mükemmel bir uyum için bu kriterin değeri 1'e yakın olmalıdır [26]. Şekil 3, önerilen modelin tahmin ettiği değerler ile gerçek değerler arasındaki ilişkiyi göstermektedir. Şekilde görüldüğü üzere, $R^{2}$ değerleri eğitim, doğrulama ve test safhaları için sırasıyla $0,9823,0,9223$ ve 0,903 olmuştur. Hesaplanan bu değerler, modelin iyi bir performansa sahip olduğunu kanıtlamış ve YSA'nın tahmin maksatlı kullanılabilir olduğunu göstermiştir.

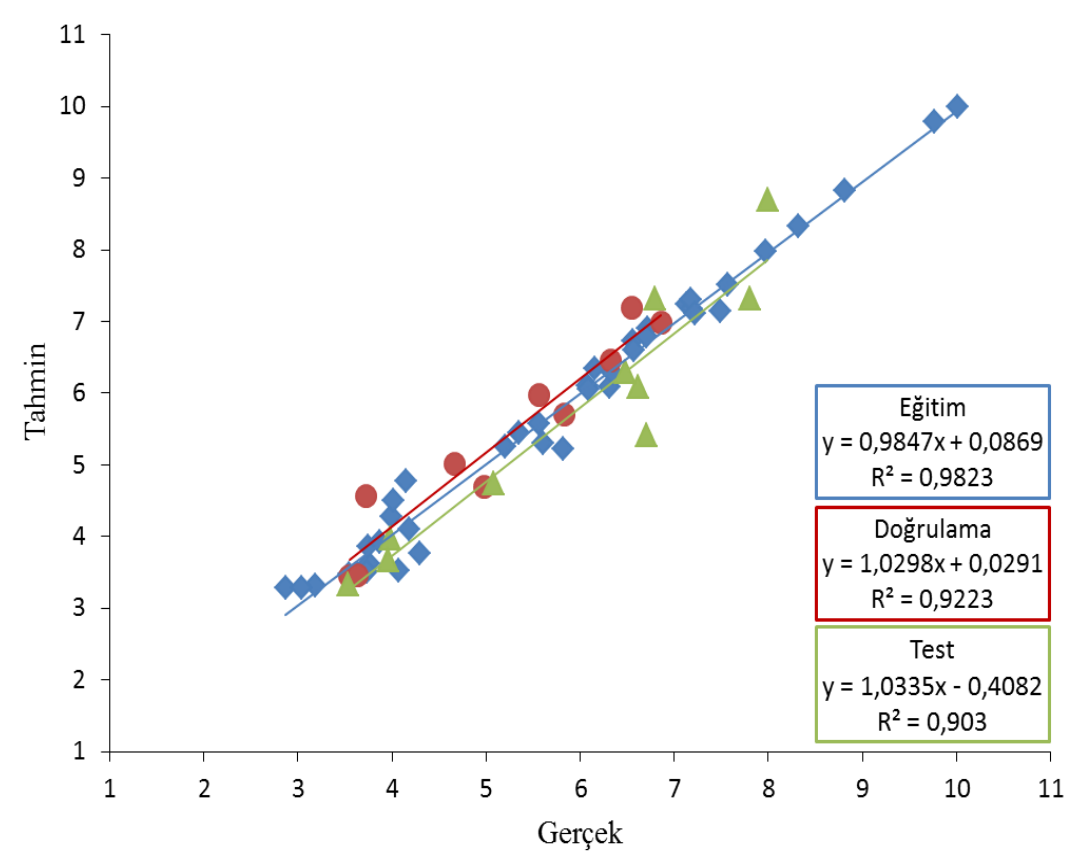

Şekil 3. Ölçülen sonuçlar ile tahmin edilen değerler arasındaki ilişki

Eğitim, doğrulama ve test setleri için tahmin edilen değerler ile ölçülen değerleri karşılaştıran grafikler Şekil 4'te verilmiştir. Bu şekil, yüzey pürüzlülüğü tahmin modelinin gerçek çıktılara yeterince yakın değerler tahmin ettiğini sergilemiştir. 
(a)

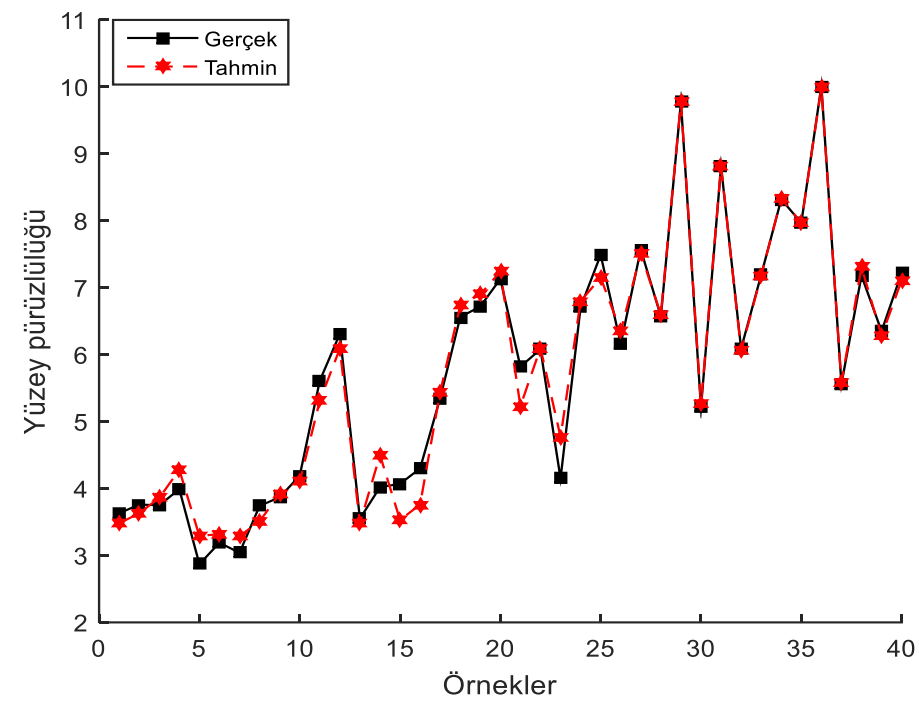

(b)

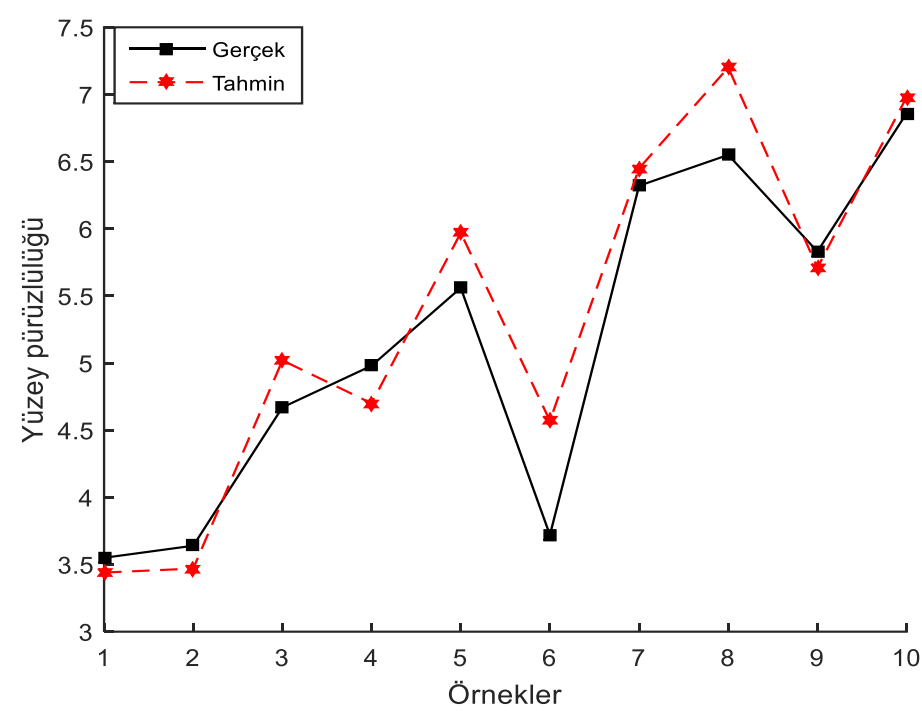

(c)

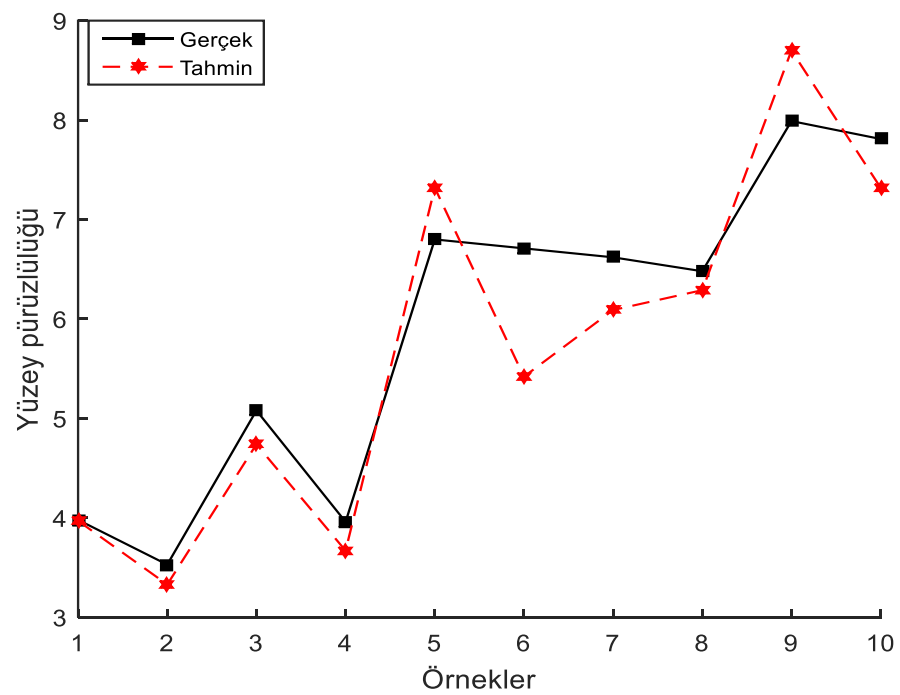

Şekil 4. Ölçülen ve tahmin edilen değerlerin karşılaştırılması: (a) eğitim, (b) doğrulama ve (c) test 
İyi eğitilen bir YSA, optimizasyon çalışmaları için ara değerleri hesaplayabilir. Bu çalışmada, odun türü ve bıçak sayısı sırasıyla sarıçam ve 2 olarak sabit tutulmuş ve kesme derinliği ile besleme hızı faktörlerinin yüzey pürüzlülüğü üzerindeki etkisi tahmin edilmiştir. Başka bir denemede ise odun türü ve bıçak sayısı sırasıyla ceviz ve 3 olarak sabitlenmiş ve kesme derinliği ile besleme hızı değiştirilmiştir. Deneysel çalışmadan elde edilmeyen Ra değerleri YSA modeli ile belirlenmiş ve Şekil 5 'te sunulmuştur.
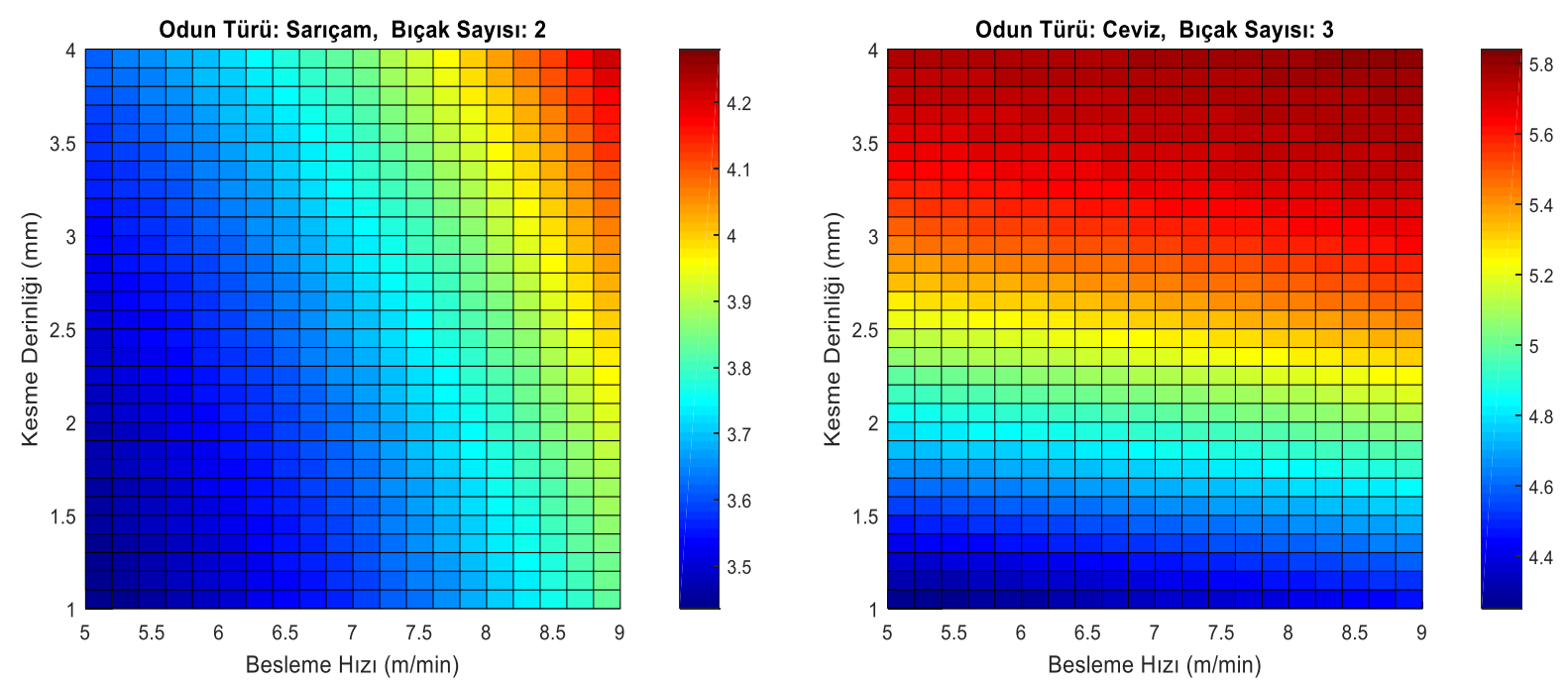

Şekil 5. Kesme derinliğinin ve besleme hızının yüzey pürüzlülüğ̈̈̈̈̈zerine etkileri

Yukarıdaki şekle göre, odunun yüzey pürüzlülüğü azalan besleme hızı ve kesme derinliği ile azalmaktadır. Bazı araştırmacılar tarafından da benzer sonuçlar bildirilmiştir [8, 9, 27, 28]. Sonuç olarak, bu araştırma çalışması işlenmiş odunun yüzey kalitesini iyileştirmek için yararlı bilgiler sunmaktadır. Tahmin modeli, farklı işleme koşullarının odunun yüzey pürüzlülüğünü nasıl etkilediğini daha iyi anlamamızı sağlamıştır.

\section{SONUC}

$\mathrm{Bu}$ çalışmada, odun türü, bıçak sayısı, besleme hızı ve kesme derinliği değişkenlerinin yüzey pürüzlülüğü üzerine olan etkileri YSA yaklaşımı ile modellenmiştir. Bahsi geçen değişkenlerin etkilerini incelemek için çok katmanlı bir sinir ağı geliştirilmiştir.

Çalışmanın sonuçları, odun yüzey pürüzlülüğü değerlerini tahmin etmek için YSA'nın oldukça uygun olduğunu göstermiştir. Model tarafından tahmin edilen yüzey pürüzlülüğü değerleri gerçek çıktılarla benzerlik göstermiştir. Test safhasındaki MAPE, RMSE ve $\mathrm{R}^{2}$ değerleri sırasıyla \% 7,27, 0,57 ve 0,903 olmuştur. Değişkenler arasındaki ilişkilerin karmaşıklığı göz önüne alındığında sonuçların tatmin edici olduğu söylenebilir.

İşleme parametrelerinin deneysel süreçte dikkate alınmayan ara değerlerine karşıllık gelen yüzey pürüzlülügüü değerleri YSA modeli ile tahmin edilmiştir. Sonuçlar göstermiştir ki, odunun yüzey pürüzlülüğü besleme hızı ve kesme derinliği azaldıkça azalmaktadır. 
Sonuç olarak bu araştırma çalışması, zaman alıcı ve maliyetli deneysel faaliyetlerde bulunmak yerine YSA tarafından tatmin edici sonuçların alınabileceğini göstermiştir.

TEȘEKKÜR: Yazarlar, çalışmada kullanılan verileri sağladığı için Dr. Selçuk Demirci'ye teşekkür etmektedir. Bu çalışma, International Forest Products Congress adlı kongrede sunulmuştur.

\section{KAYNAKLAR}

[1] C. Söğ̈̈tlü, P. Nzokou, I. Koc, R. Tutgun and N. Döngel, "The effects of surface roughness on varnish adhesion strength of wood materials," Journal of Coatings Technology and Research, vol. 13, no. 5, pp. 863-870, 2016.

[2] S. D. Sofuoğlu and A. Kurtoğlu, "Effects of machining conditions on surface roughness in planing and sanding of solid wood," Drvna Industrija, vol. 66, no. 4, pp. 265-272, 2015.

[3] L. Gurau and M. Irle, "Surface roughness evaluation methods for wood products: a review," Current Forestry Reports, vol. 3, no. 2, pp. 119-131, 2017.

[4] M. Budakci, L. Gurleyen, H. Cinar and S. Korkut, "Effect of wood finishing and planing on surface smoothness of finished wood," Journal of Applied Sciences, vol. 7, no. 16, pp. 2300-2306, 2007.

[5] B. Hendarto, E. Shayan, B. Ozarska and R. Carr, "Analysis of roughness of a sanded wood surface," International Journal of Advanced Manufacturing Technology, vol. 28, no. 7-8, pp. 775780, 2006.

[6] İ. Aydın ve G. Çolakoğlu, “Odun yüzeylerinde pürüzlülük ve pürüzlülük ölçüm yöntemleri,” Artvin Orman Fakültesi Dergisi, c. 4, s. 1, ss. 92-102, 2003.

[7] E. Csanády, E. Magoss and L. Tolvaj, Quality of machined wood surfaces, Basel: Springer International Publishing, 2015.

[8] H. Efe, S. Demirci and Y. Kilic, "Effect of the cutting direction, number of cutters, feed rate and cutting depth to the surface roughness in planning beech (Fagus orientalis Lipsky) wood," Kastamonu University Journal of Forestry Faculty, vol. 3, no. 1, pp. 77-87, 2003.

[9] I. Usta, S. Demirci and Y. Kilic, "Comparison of surface roughness of locust acacia (Robinia pseudoacacia L.) and european oak (Quercus petraea (Mattu.) Lieble.) in terms of the preparative process by planing," Building and Environment, vol. 42, no. 8, pp. 2988-2992, 2007.

[10] S. Demirci, "Effect of the number of knives, feed rate and cutting depth on surface roughness of some wood species processed with planer," Kastamonu University Journal of Forestry Faculty, vol. 13, no. 1, pp. 100-108, 2013. 
[11] A. Rolleri, F. Burgos and A. Aguilera, "Surface roughness and wettability variation: the effect of cutting distance during milling of pinus radiata wood," Drvna Industrija, vol. 67, no. 3, pp. 223$228,2016$.

[12] R. Haghbakhsh, H. Adib, P. Keshavarz, M. Koolivand and S. Keshtkari, "Development of an artificial neural network model for the prediction of hydrocarbon density at high-pressure, hightemperature conditions," Thermochimica Acta, vol. 551, pp. 124-130, 2013.

[13] I. Yildirim, S. Ozsahin and K. C. Akyuz, "Prediction of the financial return of the paper sector with artificial neural networks,” BioResources, vol. 6, no. 4, pp. 4076-4091, 2011.

[14] Ş. Özşahin, "The use of an artificial neural network for modeling the moisture absorption and thickness swelling of oriented strand board," BioResources, vol. 7, no. 1, pp. 1053-1067, 2012.

[15] C. Demirkir, Ş. Özsahin, I. Aydin and G. Colakoglu, "Optimization of some panel manufacturing parameters for the best bonding strength of plywood," International Journal of Adhesion and Adhesives, 46, pp. 14-20, 2013.

[16] S. Ozsahin, "Optimization of process parameters in oriented strand board manufacturing with artificial neural network analysis," European Journal of Wood and Wood Products, vol. 71, no. 6, pp. 769-777, 2013.

[17] S. Tiryaki, Ş. Özşahin and A. Aydın, "Employing artificial neural networks for minimizing surface roughness and power consumption in abrasive machining of wood," European Journal of Wood and Wood Products, vol. 75, no. 3, pp. 347-358, 2017.

[18] A. K. Yadav and S. S. Chandel, "Solar radiation prediction using artificial neural network techniques: a review," Renewable and Sustainable Energy Reviews, vol. 33, pp. 772-781, 2014.

[19] S. Haykin, Neural networks: a comprehensive foundation, New York: Macmillan College Publishing Company, 1994.

[20] A. M. Kalteh, "Monthly river flow forecasting using artificial neural network and support vector regression models coupled with wavelet transform," Computers \& Geosciences, vol. 54, pp. 18, 2013.

[21] G. Z. Quan, Z. Y. Zou, T. Wang, B. Liu, J. C. Li, "Modeling the hot deformation behaviors of as-extruded 7075 aluminum alloy by an artificial neural network with back-propagation algorithm," High Temperature Materials and Processes, vol. 36, no. 1, pp. 1-13, 2017.

[22] D. Z. Antanasijević, V. V. Pocajt, D. S. Povrenović, M. D. Ristić and A. A. Perić-Grujić, " $\mathrm{PM}_{10}$ emission forecasting using artificial neural networks and genetic algorithm input variable optimization," Science of the Total Environment, vol. 443, pp. 511-519, 2013.

[23] S. Tiryaki, S. Bardak and A. Aydın, "Modeling of wood bonding strength based on soaking temperature and soaking time by means of artificial neural networks," International Journal of Intelligent Systems and Applications in Engineering, vol. 4, pp. 153-157, 2016. 
[24] V. Yadav and S. Nath, "Forecasting of $\mathrm{PM}_{10}$ using autoregressive models and exponential smoothing technique," Asian Journal of Water, Environment and Pollution, vol. 14, no. 4, pp. 109$113,2017$.

[25] F. Taşpınar and Z. Bozkurt, "Application of artificial neural networks and regression models in the prediction of daily maximum $\mathrm{PM}_{10}$ concentration in Düzce, Turkey," Fresenius Environmental Bulletin, vol. 23, no. 10, pp. 2450-2459, 2014.

[26] S. Ozsahin and I. Aydin, "Prediction of the optimum veneer drying temperature for good bonding in plywood manufacturing by means of artificial neural network," Wood Science and Technology, vol. 48, no. 1, pp. 59-70, 2014.

[27] D. A. Stumbo, "Surface texture measurements for quality and production control," Forest Products Journal, vol. 10, no. 12, pp. 122-124, 1960.

[28] S. Tiryaki, A. Malkoçoğlu and Ş. Özşahin, "Using artificial neural networks for modeling surface roughness of wood in machining process," Construction and Building Materials, vol. 66, pp. 329-335, 2014. 